\title{
Desorden esquelético con malformaciones faciales en cerámico de la cultura Moche
}

\section{Skeletal disorder with facial malformations in ceramic of Moche Culture}

Correspondencia Nelson Purizaca Rosillo nelson.purizaca@upch.pe

Recibido: 06/01/2020

Arbitrado por pares

Aprobado: 26/02/2020

Citar como: Purizaca-Rosillo $N$, Correa-Trigoso DE, Gayoso $G$, Gallardo-Jugo B. Desorden esquelético con malformaciones faciales en cerámico de Cultura Moche. Acta Med Peru. 2020;37(1):74-7. doi: https://doi. org/10.35663/amp.2020.371.860
Nelson Purizaca-Rosillo,a, Denis E. Correa-Trigoso²,b, Guillermo Gayoso ${ }^{3, b}$, Bertha Gallardo-Jugo ${ }^{4, c}$

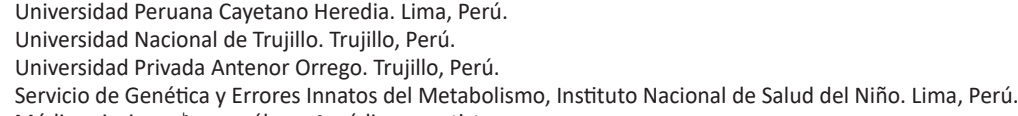

\section{RESUMEN}

La cultura Moche se desarrolló entre los años 200 y 850 d. C. en la costa norte del Perú. Sus vasijas de cerámica presentan un gran nivel de detalle, alto grado de realismo y se ha podido identificar distintas malformaciones físicas en sus representaciones. Se presenta el caso del cerámico C-00122 del Museo de Historia Natural y Cultural de la Universidad Privada Antenor Orrego (MHNC-UPAO), con un cuadro de desorden esquelético asociado a malformaciones faciales, además se plantean una serie de posibles diagnósticos etiológicos.

Palabras clave: Paleopatología; Displasias óseas; Anomalías congénitas; Perú (fuente: DeCSBIREME).

\begin{abstract}
Moche is a culture that developed between 200 y 850 AD in the Peruvian northern coast. Their pottery artefacts were crafted showing many details, they were very realistic, and different physical malformations have been identified in these pieces. We present the case of the piece C-00122 that is kept in the Natural and Cultural History Museum of Universidad Privada Antenor Orrego (NCHM-UPAO), which shows a skeletal disorder associated to some facial malformations. We discuss the case and propose different likely diagnoses.
\end{abstract}

Keywords: Paleopathology; Bone dysplasia; Congenital abnormalities; Peru (source: MeSH NLM). 


\section{INTRODUCCIÓN}

La cultura Moche es ubicada cronológicamente entre los años 200 y 850 d. C. Su área de influencia comprendió los territorios que van entre los valles de Piura (departamento de Piura) y Santa (departamento de Ancash) en la costa norte del Perú [1]. El desarrollo artístico de esta población fue de un nivel superior a las demás sociedades de los andes centrales, debido a su trabajo realista elaborado en la cerámica, siendo las divinidades, hombres, animales y escenas, dotadas de un gran nivel de realismo por parte del artesano Moche; y se considera a las vasijas de cerámica como su principal soporte de comunicación sobre las ceremonias y mitos que ellos realizaban ${ }^{[2,3]}$.

Dentro de las diferentes formas de vasijas que fueron elaboradas por los alfareros moche, destaca el tipo escultórico, por ello y por su nivel en detalle de su plasticidad escultórica se ha identificado distintas malformaciones físicas como cifosis lumbar, fisura labio palatina, siameses, pie equino varo y los síndromes de Crouzon y Seckel ${ }^{[4-7]}$.

En el presente reporte describimos las características de un cerámico de la cultura Moche que presentó múltiples malformaciones, a través de términos del Human Phenotype Ontology (HPO) y se plantean una serie de posibles diagnósticos etiológicos.

\section{REPORTE DE CASO}

Se presenta el cerámico de código C-00122 de filiación cultural Moche de la colección de material prehispánico que custodia el Museo de Historia Natural y Cultural de la Universidad Privada Antenor Orrego (MHNC-UPAO), en la ciudad de Trujillo, Perú (Figura 1). El bien cultural analizado es un cántaro escultórico antropomorfo (representación de personaje), presentó tocado, camiseta y las piernas no fueron plasmadas. El cerámico está pintado en rojo y crema, fue elaborado con la técnica del modelado y cocido en atmósfera oxidante, de dimensiones: 24 $\mathrm{cm}$ de alto, $18 \mathrm{~cm}$ de ancho y $55 \mathrm{~cm}$ de diámetro.

Se asignó un término estándar según el HPO a cada una de sus características, y posteriormente se planteó posibilidades diagnósticas. Las características se describen en la Figura 2.

\section{DISCUSIÓN}

En base al acortamiento de las extremidades y alteraciones del tórax, concluimos que hay una alteración en el desarrollo óseo por lo cual estamos ante un desorden esquelético (DE) asociado a malformaciones faciales de probable origen genético.

En la Tabla 1 se describen los principales desórdenes esqueléticos considerados como probables diagnósticos etiológicos. El síndrome de Robinow se caracteriza por presentar similares características faciales a los descritos ${ }^{[8]}$, además de acortamiento de extremidades, principalmente mesomélico y alteraciones en los genitales; sin embargo, en el cerámico C-00122 no se pudo determinar el segmento de la extremidad superior afectada ni se representaron los genitales, por lo cual no es posible afirmar que este es el diagnóstico definitivo. Este síndrome ha sido planteado anteriormente por Carod-Artal et al. en otro cerámico Moche ${ }^{[4]}$, y pese a no ser un diagnóstico definitivo, se deben buscar otros cerámicos Moche con similares características para poder saber si personas con este síndrome estuvieron presentes en la cultura Moche. Otra posibilidad diagnóstica fue la acondroplasia debido al acortamiento de las extremidades y algunas características faciales ${ }^{[9]}$, sin embargo, no se asocia a fisura labial como el cerámico C-00122.

Otros diagnósticos menos probables fueron síndrome JarchoLevin por las alteraciones a nivel costal ${ }^{[10]}$, sin embargo, no hay afectación de miembros superiores como en el cerámico de estudio y síndrome Aarskog ${ }^{[11]}$, por las características faciales, no obstante, no se caracteriza por acortamiento en las extremidades, además en el cerámico no hay una aparente disminución del tamaño de la mano como es frecuente en este síndrome.

La representación de personas con enfermedades genéticas en los cerámicos Moche está documentada ${ }^{[6,12]}$, pero ninguno corresponde a las características descritas en este cerámico. Aunque no es posible tener el diagnóstico etiológico definitivo, creemos que el nivel de detalle de la cerámica moche, nos permite afirmar que las DE estuvieron presentes desde el periodo Moche.

Los DE son de origen genético y hereditario ${ }^{[13]}$. Es importante definir si el tipo específico de DE presenta herencia autosómica recesiva, por la posible presencia de personas portadoras desde el periodo Moche a la actualidad. Por ejemplo, actualmente existen poblaciones descendientes de la cultura Moche, como Mórrope ${ }^{[14]}$, con baja tasa de migración dónde estos genes patogénicos podrían estar concentrados (consanguinidad territorial) aumentando la probabilidad de aparición de estos cuadros.

Dentro de las limitaciones, la principal fue que en el cerámico no se representaron algunas estructuras como los miembros inferiores y los genitales, necesarios para aproximar un diagnóstico con mayor precisión. Además, no es posible determinar la historia familiar del individuo representado, que es fundamental para determinar el tipo de herencia.

En conclusión, los desórdenes esqueléticos existieron durante la época de la cultura Moche. Es importante realizar evaluaciones de mayor cantidad de cerámicos para poder determinar los tipos específicos de DE y sus tipos de herencia.

Contribuciones de autoría: Los autores declaran haber realizado contribuciones significativas a la concepción y diseño del manuscrito, la recolección de los datos, redacción y revisión crítica del contenido del manuscrito, la aprobación final de la versión que se publicará y asumen la responsabilidad de todos los aspectos del manuscrito. 


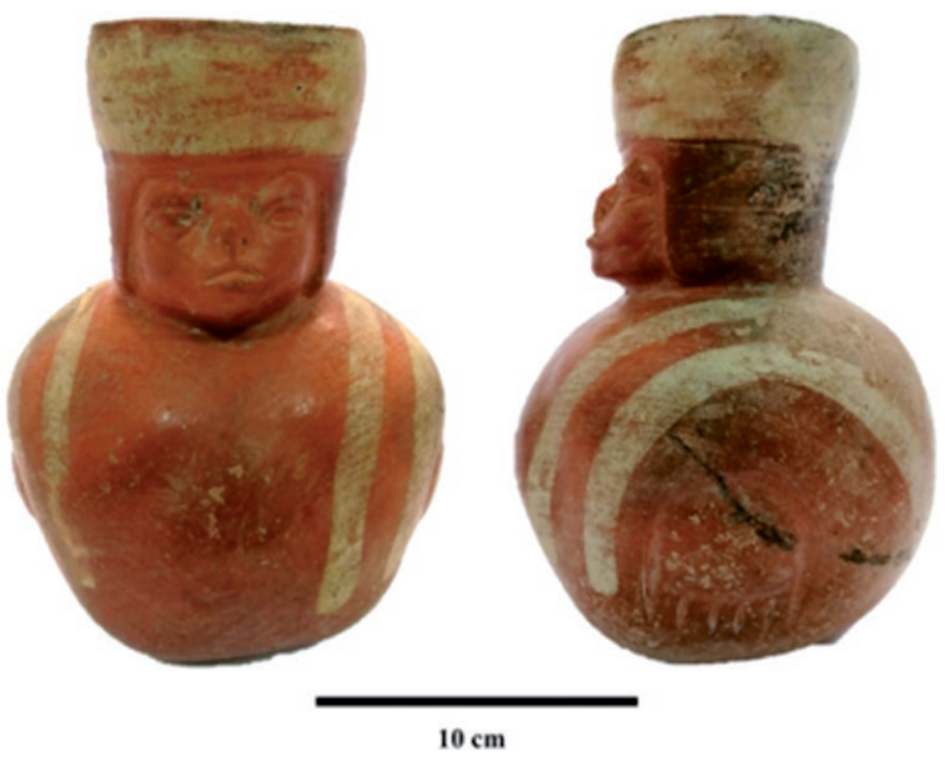

Figura 1. Pieza de cerámica C-00122 del Museo de Historia Natural y Cultural de la Universidad Privada Antenor Orrego.

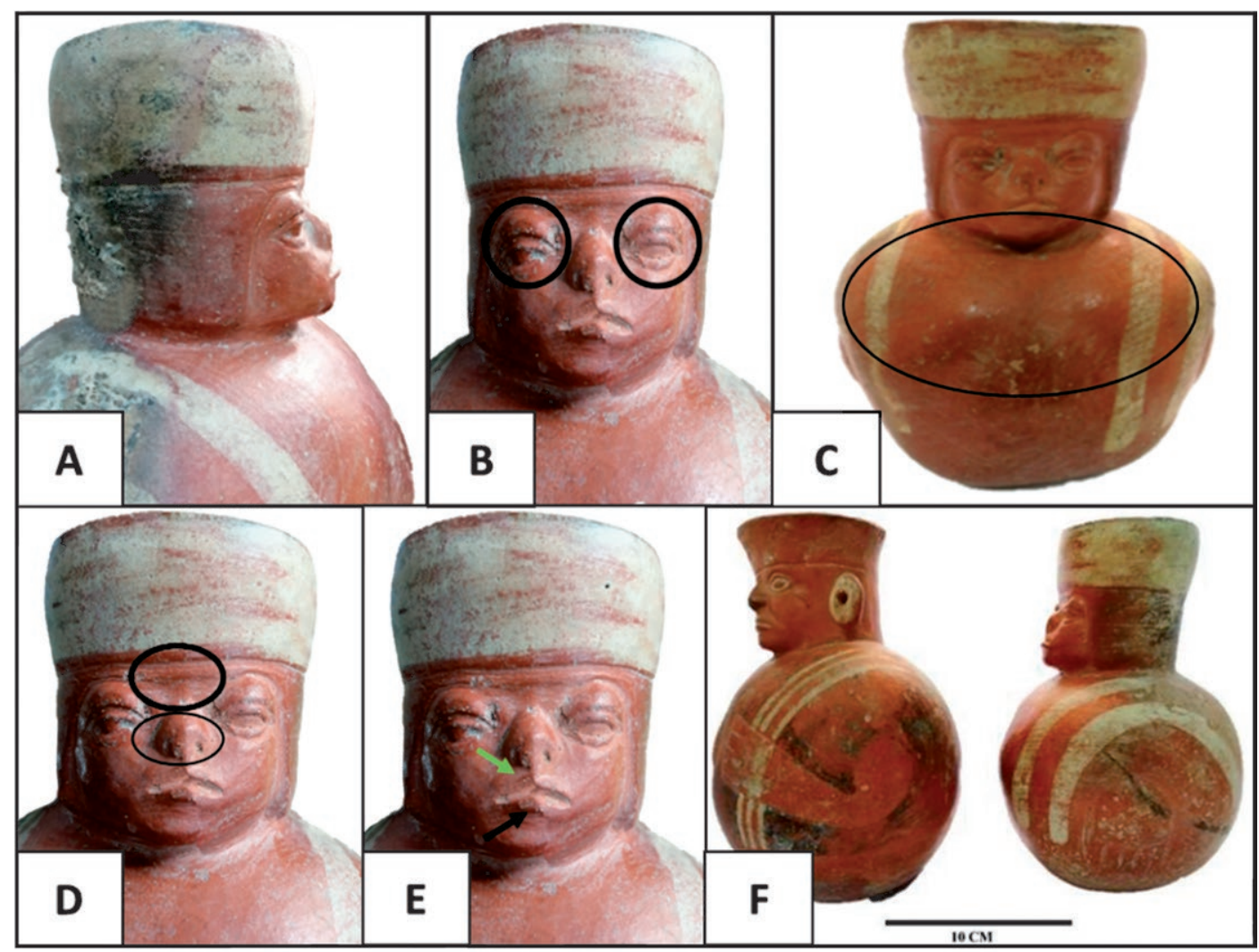

Figura 2. A. Mitad superior de la cara en una posición posterior respecto a la mitad inferior, con aumento de la concavidad de la cara (Midface retrusion - HPO: 0011800). B. Región ocular prominente (Proptosis HPO:0000520). C. Falta de simetría entre la mitad izquierda y derecha del tórax (Asymmetry of the thorax - HPO: 0001555). D. Posición posterior de la raíz nasal (Depressed nasal bridge HPO:0005280). Punta de la región correspondiente a la nariz hacia arriba con las fosas nasales orientadas hacia delante. (Anteverted nares - HPO: 0000463). Disminución de la longitud del puente de la nariz hasta su punta nasal (Short nose - HPO: 0003196). E. Interrupción de la continuidad en labio superior (flecha verde) (Cleft upper lip HP:0000204). Aumento del grosor del labio inferior (flecha negra) (Thick lower lip vermilion HPO:0000179). F. Comparación con otro cerámico moche de similares características, donde se evidencia que el individuo representado en el cerámico C-00122 presenta acortamiento de extremidad superior. 
Tabla 1. Principales diagnósticos diferenciales para el individuo representado en el cerámico C-00122 y sus características reconocibles ectoscópicamente

\begin{tabular}{|c|c|c|c|c|}
\hline $\begin{array}{l}\text { Diagnóstico } \\
\text { etiológico }\end{array}$ & $\begin{array}{l}\text { Tipo de } \\
\text { herencia }\end{array}$ & Características concordantes & $\begin{array}{c}\text { Característica no } \\
\text { concordantes }\end{array}$ & $\begin{array}{l}\text { Características } \\
\text { faltante }\end{array}$ \\
\hline \multirow{6}{*}{$\begin{array}{l}\text { Síndrome de } \\
\text { Robinow }\end{array}$} & & Narinas antevertidas & \multirow{6}{*}{$\begin{array}{l}\text { Proptosis } \\
\text { Tórax asimétrico }\end{array}$} & \\
\hline & & Depresión de puente nasal & & Micropene \\
\hline & $A D$ & Nariz corta & & Criptorquidea \\
\hline & AR & Retracción media facial & & Braquidactilia \\
\hline & & Fisura labial & & \\
\hline & & Acortamiento de brazos & & \\
\hline \multirow{4}{*}{ Acondroplasia } & \multirow{4}{*}{$A D$} & \multirow{4}{*}{$\begin{array}{l}\text { Retracción media facial } \\
\text { Acortamiento de brazos } \\
\text { Depresión de puente nasal }\end{array}$} & \multirow{4}{*}{$\begin{array}{c}\text { Proptosis } \\
\text { Fisura Labial } \\
\text { Nariz Corta } \\
\text { Narinas antevertidas } \\
\text { Tórax asimétrico }\end{array}$} & Frente prominente \\
\hline & & & & Cifosis toracolumbar \\
\hline & & & & Braquidactilia \\
\hline & & & & $\begin{array}{c}\text { Estatura corta } \\
\text { desproporcionada }\end{array}$ \\
\hline
\end{tabular}

AD: Autosómico Dominante. AR: Autosómico Recesivo.

Potenciales conflictos de interés: Los autores declaran no tener conflictos de intereses.

Fuente de financiamiento: Autofinanciado.

\section{ORCID:}

Nelson Purizaca-Rosillo, https://orcid.org/0000-0003-4773-328X Bertha Gallardo-Jugo, https://orcid.org/0000-0002-4747-9325 Denis E. Correa-Trigoso, https://orcid.org/0000-0002-1262-1432 Guillermo Gayoso, https://orcid.org/0000-0001-8173-365X

\section{REFERENCIAS BIBLIOGRÁFICAS}

1. Castillo L, Uceda S. The Mochicas. En: Silverman H, Isbell W. The Handbook of South America Archaeolog. Nueva York: Springer; 2008. p.707-29.

2. Donnan C. Moche Art of Peru: Pre-Columbian symbolic communication. Los Angeles: Museum of Cultural History, University of California; 1978.

3. Donan, C. Moche portraits from ancient Peru. First Edition. Austin: University of Texas Press; 2004.

4. Carod-Artal FJ, Vázquez CB. Malformaciones y parálisis faciales en la cerámica de las culturas precolombinas Moche y Lambayeque. Neurologia. 2006;21(6):297-303.

5. Larco R. Los Mochicas Tomo II. Lima: Museo Arqueológico Rafael Larco Herrera, Servicios Editoriales del Perú; 2001.
6. Correa-Trigoso D. Presencia de paleopatologías en las representaciones mochica: Un estudio de la colección cerámica del Museo Larco. Horizonte de la Ciencia.2017;7(12):43-60.

7. Klein E, Gallardo B, Chávez M, Abarca-Barriga H. Atlas de dismorfología pediátrica. Primera edición. Lima: Instituto Nacional de Salud del Niño; 2012.

8. Beiraghi S, Leon-Salazar V, Larson BE, John MT, Cunningham $M L$, Petryk $A$, et al. Craniofacial and intraoral phenotype of Robinow syndrome forms. Clin Genet. 2011;80(1):15-24. doi: 10.1111/j.1399-0004.2011.01683.x

9. Daugherty A. Achondroplasia: Etiology, Clinical Presentation, and Management. Neonatal Netw. 2017;36(6):337-42. doi: 10.1891/0730-0832.36.6.337

10. Kurup PM, Tanigasalam V, Bhat BV. Spondylocostal Dysostosis (Jarcho Levin Syndrome). Indian J Pediatr. 2018;85(6):486. doi: 10.1007/s12098-017-2549-0

11. Ahmed A, Mufeed A, Ramachamparambathu AK, Hasoon U. Identifying Aarskog Syndrome. J Clin Diagn Res. 2016;10(12):ZD09ZD11. doi: 10.7860/JCDR/2016/22180.8982

12. Pachajoa $H$, Rodríguez $C$. Defectos congénitos y síndromes genéticos en el arte de las sociedades Tumaco-Tolita y Moche. primera edición. Cali: Universidad ICESI; 2017.

13. Mortier GR, Cohn DH, Cormier-Daire V, Hall C, Krakow D, Mundlos $S$, et al. Nosology and classification of genetic skeletal disorders: 2019 revision. Am J Med Genet A. 2019;179(12):2393-419. doi: 10.1002/ajmg.a.61366

14. Miranta L. Murrup. Último pueblo Mochica. Primera edición. Lima: Editorial Super Gráfica; 2014. 